\title{
HIGHLY ACCURATE CALCULATION OF THE REAL AND COMPLEX EIGENVALUES OF ONE-DIMENSIONAL ANHARMONIC OSCILLATORS
}

\author{
Francisco Marcelo Fernández*, Javier Garcia
}

\author{
INIFTA (UNLP, CCT La Plata-CONICET), Blvd. 113 S/N, Sucursal 4, Casilla de Correo 16, 1900 La Plata, \\ Argentina \\ * corresponding author: fernande@quimica.unlp.edu.ar
}

\begin{abstract}
We draw attention on the fact that the Riccati-Padé method developed some time ago enables the accurate calculation of bound-state eigenvalues as well as of resonances embedded either in the continuum or in the discrete spectrum. We apply the approach to several one-dimensional models that exhibit different kind of spectra. In particular we test a WKB formula for the imaginary part of the resonance in the discrete spectrum of a three-well potential.
\end{abstract}

KEYWORDS: anharmonic oscillators; bound states; resonances; Riccati-Padé method; WKB asymptotic expression.

\section{INTRODUCTION}

In a recent paper Gaudreau et al[1] proposed a method for the calculation of the eigenvalues of the Schrödinger equation for one-dimensional anharmonic oscillators. In their analysis of some of the many approaches proposed earlier with that purpose they resorted to expressions of the form: "However, the existing numerical methods are mostly case specific and lack uniformity when faced with a general problem." "As can be seen by the numerous approaches which have been developed to solve this problem, there is a beautiful diversity yet lack of uniformity in its resolution. While several of these methods yield excellent results for specific cases, it would be favorable to have one general method that could handle any anharmonic potential while being capable of computing efficiently approximations of eigenvalues to a high pre-determined accuracy." "Various methods have been used to calculate the energy eigenvalues of quantum anharmonic oscillators given a specific set of parameters. While several of these methods yield excellent results for specific cases, there is a beautiful diversity yet lack of uniformity in the resolution of this problem." The authors put forward an approach that they termed double exponential Sinc collocation method (DESCM) and reported results of remarkable accuracy for a wide variety of problems. In fact they stated that "In the present work, we use this method to compute energy eigenvalues of anharmonic oscillators to unprecedented accuracy" which may perhaps be true for some of the models chosen but not for other similar examples. For example, in an unpublished article Trott [2] obtained the ground-state energy of the anharmonic oscillator with potential $V(x)=x^{4}$ with more than 1000 accurate digits. His approach is based on the straightforward expansion of the wavefunction in a Taylor series about the origin.

One of the methods mentioned by Gaudreau et al[1] is the Riccati-Padé method (RPM) based on a rational approximation to the logarithmic derivative of the wavefunction that satisfies a well known Riccati equation 3 , 4]. In their brief analysis of the RPM the authors did not mention that this approach not only yields the bound-state eigenvalues but also the resonances embedded in the continuum[5]. What is more, the same RPM quantization condition, given by a Hankel determinant, produces the bound-state eigenvalues, the resonances embedded in the continuum as well as some kind of strange resonances located in the discrete spectrum of some multiple-well oscillators [6]. It is not clear from the content of [1] whether the DESCM is also suitable for the calculation of such complex eigenvalues.

The accuracy of the calculated eigenvalues not only depends on the chosen method but also on the available computation facilities and on the art of programming. For this reason the comparison of the accuracy of the results reported in a number of papers spread in time should be carried out with care.

The purpose of this paper is two-fold. First, we show that the RPM can in fact yield extremely accurate eigenvalues because it exhibits exponential convergence. To that end it is only necessary to program the quantization condition in an efficient way in a convenient platform. Second, we stress the fact that the RPM yields both real and complex eigenvalues with similar accuracy through the same quantization condition. More precisely: it is not necessary to modify the algorithm in order to obtain such apparently dissimilar types of eigenvalues that are associated to different boundary conditions of the eigensolution.

In section 2 we outline the RPM for even-parity potentials. In section 3 we apply this approach to some of the examples discussed by Gaudreau et al[1] and obtain eigenvalues with remarkable accuracy. In this section we also calculate several resonances supported by anharmonic oscillators that were not taken into account by those authors. 
We consider examples of resonances embedded in the continuous as well as in the discrete spectrum. Finally, in section 4 we summarize the main results and draw conclusions.

\section{The Riccati-Padé Method}

The dimensionless Schrödinger equation for a one-dimensional model reads

$$
\psi^{\prime \prime}(x)+[E-V(x)] \psi(x)=0,
$$

where $E$ is the eigenvalue and $\psi(x)$ is the eigenfunction that satisfies some given boundary conditions. For example, $\lim _{|x| \rightarrow \infty} \psi(x)=0$ determines the discrete spectrum and the resonances are associated to outgoing waves in each channel (for example, $\psi(x) \sim A e^{i k x}$ ). In this paper we restrict ourselves to anharmonic oscillators with even-parity potentials $V(-x)=V(x)$ to facilitate the comparison with the results reported by Gaudreau et al[1] but it should be taken into account that the approach applies also to no non-symmetric potentials [7].

In order to apply the RPM we define the regularized logarithmic derivative of the eigenfunction

$$
f(x)=\frac{s}{x}-\frac{\psi^{\prime}(x)}{\psi(x)},
$$

that satisfies the Riccati equation

$$
f^{\prime}(x)+\frac{2 s f(x)}{x}-f(x)^{2}+V(x)-E=0,
$$

where $s=0$ or $s=1$ for even or odd states, respectively. If $V(x)$ is a polynomial function of $x$ or it can be expanded in a Taylor series about $x=0$ then one can also expand $f(x)$ in a Taylor series about the origin

$$
f(x)=x \sum_{j=0}^{\infty} f_{j}(E) x^{2 j} .
$$

On arguing as in earlier papers we conclude that we can obtain approximate eigenvalues to the Schrödinger equation from the roots of the Hankel determinant

$$
H_{D}^{d}(E)=\left|\begin{array}{cccc}
f_{d+1} & f_{d+2} & \cdots & f_{d+D} \\
f_{d+2} & f_{d+3} & \cdots & f_{d+D+1} \\
\vdots & \vdots & \ddots & \vdots \\
f_{d+D} & f_{d+D+1} & \cdots & f_{d+D-1}
\end{array}\right|=0
$$

where $D=2,3, \ldots$ is the dimension of the determinant and $d$ is the difference between the degrees of the polynomials in the numerator and denominator of the rational approximation to $f(x)[6$. In those earlier papers we have shown that there are sequences of roots $E^{[D, d]}, D=2,3, \ldots$ of the determinant $H_{D}^{d}(E)$ that converge towards the bound states and resonances of the quantum-mechanical problem. We have at our disposal a set of sequences for each value of $d$ but it is commonly sufficient to choose $d=0$. For this reason, in this paper we restrict ourselves to the sequences of roots $E^{[D]}=E^{[D, 0]}$ (unless stated otherwise).

In this paper we are concerned with anharmonic-oscillator potentials of the form

$$
V(x)=\sum_{j=1}^{K} v_{j} x^{2 j} .
$$

The spectrum is discrete when $v_{K}>0$ and continuous when $v_{K}<0$. In the latter case there may be resonances embedded in the continuous spectrum which are complex eigenvalues. The real part of any such eigenvalue is the resonance position and the imaginary part is half its width $\Gamma(|\Im E|=\Gamma / 2)$.

\section{EXAMPLES}

Four examples chosen by Gaudreau et al[1] are quasi-exactly solvable problems; that is to say, one can obtain exact solutions for some states:

$$
\begin{aligned}
V_{1}(x) & =x^{2}-4 x^{4}+x^{6} & E_{0} & =-2 \\
V_{2}(x) & =4 x^{2}-6 x^{4}+x^{6} & E_{1} & =-9 \\
V_{3}(x) & =\frac{105}{64} x^{2}-\frac{43}{8} x^{4}+x^{6}-x^{8}+x^{10} & E_{0} & =\frac{3}{8} \\
V_{4}(x) & =\frac{169}{64} x^{2}-\frac{59}{8} x^{4}+x^{6}-x^{8}+x^{10} & E_{1} & =\frac{9}{8} .
\end{aligned}
$$


The RPM yields the exact result for all these particular cases because in all of them the logarithmic derivative $f(x)$ is a rational function of the coordinate. The Hankel determinants of lowest dimension for each case are:

$$
\begin{aligned}
& H_{2}^{0}(E)=\frac{1}{4725}(E+2)\left(E^{5}-2 E^{4}-23 E^{3}-602 E^{2}+1030 E-1412\right), \\
& H_{2}^{0}(E)=\frac{1}{4465125}(E+9)\left(E^{5}-9 E^{4}-187 E^{3}-8217 E^{2}+78336 E-348624\right), \\
& H_{3}^{0}(E)=\frac{1}{3189612751764848640000}(8 E-3)\left(8589934592 E^{11}+3221225472 E^{10}\right. \\
& -1887235473408 E^{9}-399347250364416 E^{8}-1634745666502656 E^{7} \\
& +10770225531715584 E^{6}-836065166572191744 E^{5}-905684630058491904 E^{4} \\
& +5197219286067104256 E^{3}-2944302537136698432 E^{2} \\
& -12283878786837315912 E+22452709866105906693), \\
& H_{3}^{0}(E)=\frac{1}{431028319209742820966400000}(8 E-9)\left(8589934592 E^{11}+9663676416 E^{10}\right. \\
& -5569096187904 E^{9}-2064531673055232 E^{8}-15362232560910336 E^{7} \\
& +158709729905344512 E^{6}-23752960275863896064 E^{5}-84068173973645402112 E^{4} \\
& +2318080070178601634304 E^{3}-6274577633554290840768 E^{2} \\
& -75410626140297229262472 E+655367638076442656931879),
\end{aligned}
$$

respectively. It is clear that the second factor of each Hankel determinant yields the exact eigenvalue of the corresponding model in equation (7).

As a nontrivial example we consider the quartic anharmonic oscillator

$$
V(x)=x^{2}+\lambda x^{4}
$$

Gaudreau et al [1] calculated the ground state for $\lambda=1$ with remarkable accuracy. The RPM also enables great accuracy because of its exponential convergence. For example, with determinants of dimension $D \leq 623$ we obtained

$$
\begin{array}{rl}
E_{0}=1 & 39235164153029185565750787660993418460006671122083408890634932387756743187564652 \\
& 85909735634677917591211513753417388174455516240463837130438178697370013460935168 \\
& 15484208574889656901800305541236648743218953435715417409382624057229519998568711 \\
& 18140968922702273638169811112603107034293861341959645684859182914634898518858148 \\
& 63025469392145221031177208948219643654580541741801366088701870825264349698158700 \\
& 82340760759574319226851138960019685449394982096240756162094619633463447377455701 \\
& 49211492623468905916373385630626814055709925106270580909505786666030935831448351 \\
& 97352905560061049224302849821825415119194035000689109989896675454979833183805654 \\
& 19975466162573031052729404581567529262538228672118076018319975294595611113245756 \\
& 78445653018419567798509749315372254188588216960225999726980950846580656370213654 \\
& 47651793869049904755455309191949465274340562585980971938979595684138772300267900 \\
& 68177673277845708654477245631366268184519934644126051969150124972306172724393638 \\
& 74511499751517142498813649966422950045954851519165072488133686158144218817306000 \\
& 39773536840117104637678735672726392478420532548924901523470626991951440934018875 \\
& 83071929546817823113125377471312004221881276679422460872268510606766179549130792 \\
& 640798558850522732484547554994100518213983,
\end{array}
$$

which is considerably more accurate than the result reported by those authors. Such an accuracy is unnecessary but it clearly shows the stability and remarkable rate of convergence of the RPM.

For some approaches the pure quartic oscillator

$$
V(x)=x^{4}
$$


may be more demanding than the oscillator treated previously. However, this is not the case for the RPM that yields

$E_{0}=1.06036209048418289964704601669266354551520872852829779332162452416959435630443444$ 21126896299134671720351054624435858252558087980821029314701317683637238249357892 26246004708175446960141637488417282256290593575779088806178879026360154939569027 51961489200942934873584409442694897901213971464290951923352453382834703350575761 51120257039888523720240221842110308657373109139891545365841031116794058335486020 09227440069631126702388622971429699610592155832226671376935508673610000831830027 51792623357391390621361807764985969618149941279280927284070795610604240722946809 94913627572927387279136890279842472226217169444889547513704380684054391877877295 32342458274372543178323190603810687416044034374530146847272813918612940470431034 01351071607110353008929823272542766151898695056504716025275608952626219102568822 00964410287815640052705292932405076382650282591122477362538471854714402572285438 48529745045857097828402490669995704768445877091762029124375273254907211643344023 02947306923981908956853745359884460160202313291933059395869304916644281633946163 32428700242614612377430099522342042085977356901535654168502308941851348795734106 58547971946759646679661346762885864379526545195605682867159583388847434670120422 42071491874787103842957338913898524589402226347126961769965604409311709985471606 46641857421281143028818111495112214843140887121662059313076923418022298272468836 26045356507913236221596486925870033200274440968806404623978817839469837807048268 60217427219460350750696191658224983009606134572666392863592217643534013718920448 14846483730289412529638634402446954353934473733433447707230478215508820964235121 06900382833900237848230939194834

from determinants of dimension $D \leq 806$. We think that present result is more accurate than the one reported by Trott[2], the discrepancy being in his last 9 figures. In the case of the quartic anharmonic oscillators (6) and $(12)$ it was proved that $E_{0}^{[D, 0]}$ and $E_{0}^{[D, 1]}$ are lower and upper bounds to the actual eigenvalue, respectively [3, 4]. In order to verify the accuracy of present calculation we verified that both bounds agreed to the last digit.

As stated in the introduction, the RPM yields not only the bound states but also the resonances. For example, in the case of the anharmonic oscillator $(12$ with $\lambda=-0.1$ (inverted double well) we obtain

$\Re E=0.90067290409201502480472168921028775830460331662203069831718516924034871463944702$ 49616726790089628268825293776377464732577257777502973177491351445742473385877709 26803971267801469755869917579845522512000205420137720995176766371327034480743071 07713982008790873985646991861751535214021599017867320106022879739539457665858699 75562973189279258249081617920321264175746331544068756411634377154244333590011287 04230510965330925907792495147669628533284309302211234477027796391708328562113084 41726909573060442238866068277795715277616928060425810097530790289082978267983213 67842682657484596201757300110536533702699585361715680944542285361299988968687820 12308852365127364727689632938045419946222831027030494463529154344400824268783119 49185729315006099566578108828241145777722487108371656443716078796497379465206234 879130342123770426063990565158077971857506099342729619

and

$|\Im E|=0.00669328087580013026927187508131824112294989432621696735893314097282610915605850$ 43018393541639674627243621481357409744100069700186351017147154410094249647120952 59566361194259386325794519366933621549298669570727785778446401403144312336955986 73980375283541840546888210884788662488801718718713379746362368368461205368517345 68189777241623332803606777032301489912446298837896455581815166460445660555875128 54363903733935667870354171283504808639418360504429532217074896601384341982511525 91876064523830978322014707732840778218698514295641443748495983816659272161010695 96118632431428217783653913815974284318203202992357388742962705328614872135008968 45311943228106834110088574337059948926573991903248493789402558550272582426356810 29615386184966870885537480832794653421556685850226225141032328397825281636792728 913688704017585002638602666568373554869393536520227140 


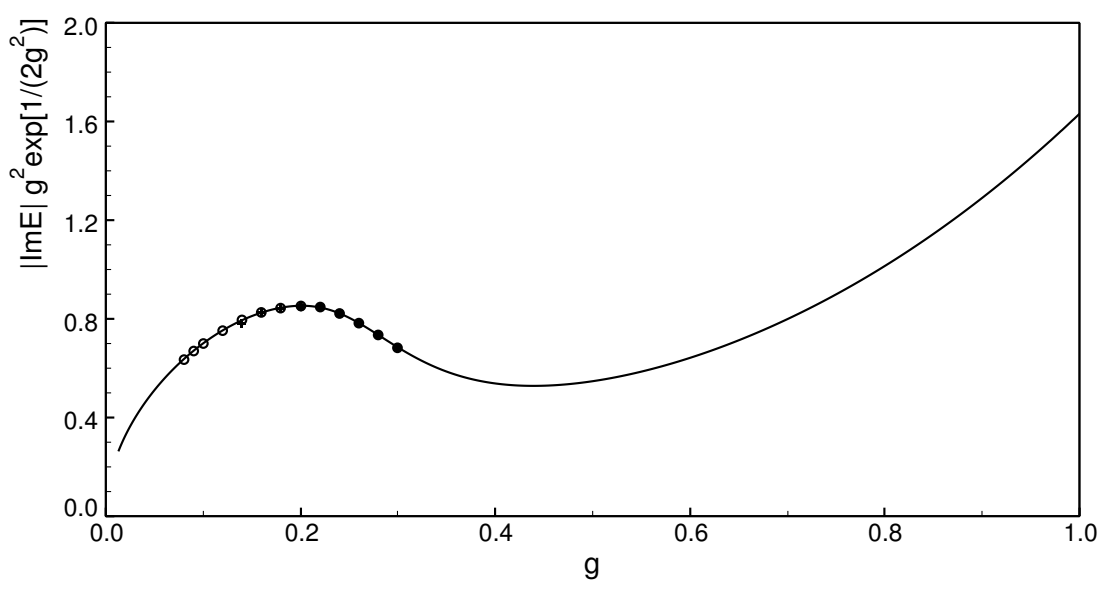

Figure 1. Present calculation of $|\Im E| g^{2} \exp \left[1 /\left(2 g^{2}\right)\right]$ for the oscillator 14 (solid line) and the results of Benassi et al 8 . (filled circles), Killingbeck [9] (crosses) and Fernández [6] (empty circles).

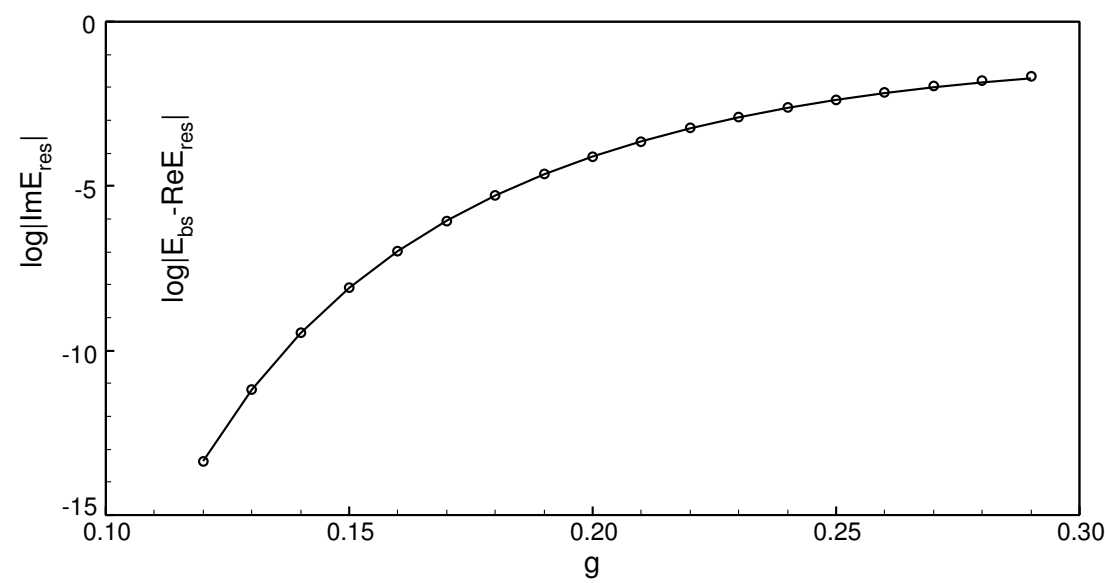

Figure 2. $\log \left|\Im E_{\text {res }}\right|$ (circles) and $\log \left|\Re E_{\text {res }}-E_{\mathrm{bs}}\right|$ (solid line) vs. $g$ for the oscillator 14 with $k=1$.

with determinants of dimension $D \leq 429$. As far as we know this resonance was not calculated with such an accuracy before.

A most interesting example is given by

$$
V(x)=x^{2}\left(1-g^{2 k} x^{2 k}\right)^{2}, \quad k=1,2, \ldots
$$

It is a three-well potential with minima $V\left(x_{\mathrm{m}}\right)=0$ at $x_{\mathrm{m}}=-1 / g, 0,1 / g$. Since $V(x)$ behaves asymptotically as $g^{4 k} x^{4 k+2}$ when $|x| \rightarrow \infty$ then one expects only bound states with positive eigenvalues when $g$ is real. However, Benassi et al 8 proved that this family of potentials supports complex eigenvalues with all the properties of actual resonances. They calculated the lowest resonance for $k=1$ and several values of $g$ and compared the imaginary part with the WKB transmission coefficient through the barrier $\left|\Im E_{\mathrm{WKB}}\right| \sim A g^{-2} e^{-1 /\left(2 g^{2}\right)}$. Later Killingbeck $[9$ ] and Fernández [6] calculated this resonance more accurately and for smaller values of $g$ and showed that $|\Im E| g^{2} e^{1 /\left(2 g^{2}\right)}$ does not exhibit a uniform behaviour as suggested by the earlier calculation of Benassi et al 8 . In particular, Killingbeck $\left[9\right.$ suggested that $|\Im E| g^{2} e^{1 /\left(2 g^{2}\right)}$ exhibits a maximum. In this paper we calculated $\Im E$ even more accurately and for smaller values of $g$ and present results, shown in Figure 1 suggest that the conjecture that "the imaginary part of the resonance behaves as the WKB transmission coefficient through the barrier" may not be correct. The results of Benassi et al[ 8 for a shorter interval of $g$ (also shown in the figure) give the impression that $|\Im E|$ has already reached the asymptotic behaviour $A g^{-2} e^{-1 /\left(2 g^{2}\right)}$ which is not the case. The figure also shows the earlier results of Killingbeck 9 and Fernández $[6$. A straightforward least-squares fitting of present results suggests that the correct behaviour is $|\Im E| \sim A g^{-3 / 2} e^{-1 /\left(2 g^{2}\right)}$.

The lowest bound state $E_{\mathrm{bs}}$ and the real part of the lowest resonance $\Re E_{\text {res }}$ approach each other as $g \rightarrow 0$ in such a way that $\left|E_{\mathrm{bs}}-\Re E_{\mathrm{res}}\right|$ is of the order of $\left|\Im E_{\mathrm{res}}\right|$. This fact is clearly shown in Figure 2 , 
The RPM enables us to calculate the bound states and resonances quite accurately. In what follows we show some of them for the potential 14 with $k=1$ and $g=0.2$.

By means of the RPM for even solutions and from determinants of dimension $D \leq 775$ we have estimated

$$
\begin{aligned}
E_{\mathrm{bs}}= & 0.93247629196422125071328330705170258832085891016425094045219553044054139703731261 \\
& 50649025687960895922690251875547008545901420481160447985150327460757263156945963 \\
& 43111670051681020512461078632259474114210076408768061413506747150659467931140457 \\
& 73553564279657808191641005760522714953011384832488596806112996385064780658000126 \\
& 21383561744399262787670426442500032348267656012265260808541171888390190812639425 \\
& 68677114253841158525832058185894866049673942610420369468954266459806798388982945 \\
& 52842394543958516825976072367841054683416775046265615981244952016961280615722797 \\
& 48522242230626330783391577288128021207208653859352779699723097379217744729225892 \\
& 87271537226473008063005886824272445164895449164007979447482255960907744830950818 \\
& 89615946058064623691858375392064608307256038193215181635793814371703342844510929 \\
& 37725091255795633498661026822076286385619429762725138253425155067910751071052934 \\
& 27678838734764019823945574715117575021473073907215347869490374168192236026830615 \\
& 21556243352973248410809257466846919735225097807676751284064233765380645890401273 \\
& 18619105202
\end{aligned}
$$

Analogously for the lowest resonance and from determinants of dimension $D \leq 691$ we obtained:

$$
\begin{aligned}
\Re E_{\mathrm{res}}= & 0.93255571582477452179676759062168990966452649573421385221674869167644974697878297 \\
& 48288966600919521327429371760006946990662957241091484006768849519862253721798569 \\
& 03644092281642425061693541023975965045234361601035467225031110683747763474057010 \\
& 51766932213808285413600743099773501734379824559724078809842601314254466796141742 \\
& 91619009303111780839333634172120375607438466877056145373073135249510667485570624 \\
& 89027647576289516788138371419308234504770062385126389060959383615011639231234684 \\
& 57211692027452357322762788434499112499793762153016569474982638402568272061044967 \\
& 57607047286711883739177838606163003503292340210633885558943987330696955212335784 \\
& 93874116286488958371712887316534334151628894506338250698992826979320533720965089 \\
& 88556846872196309982663487612817769715053392472202304048467020861359787434424925 \\
& 59863763422430231269302968797027663315298386727326660998017953973558253873774320 \\
& 28276057502051489621320017464691543602109098291291138852305607511705280075050547 \\
& 31063488489167352701182508545578769602211943958747945495907096406141902643986905 \\
& 961993842355299216457009181864846232
\end{aligned}
$$

and

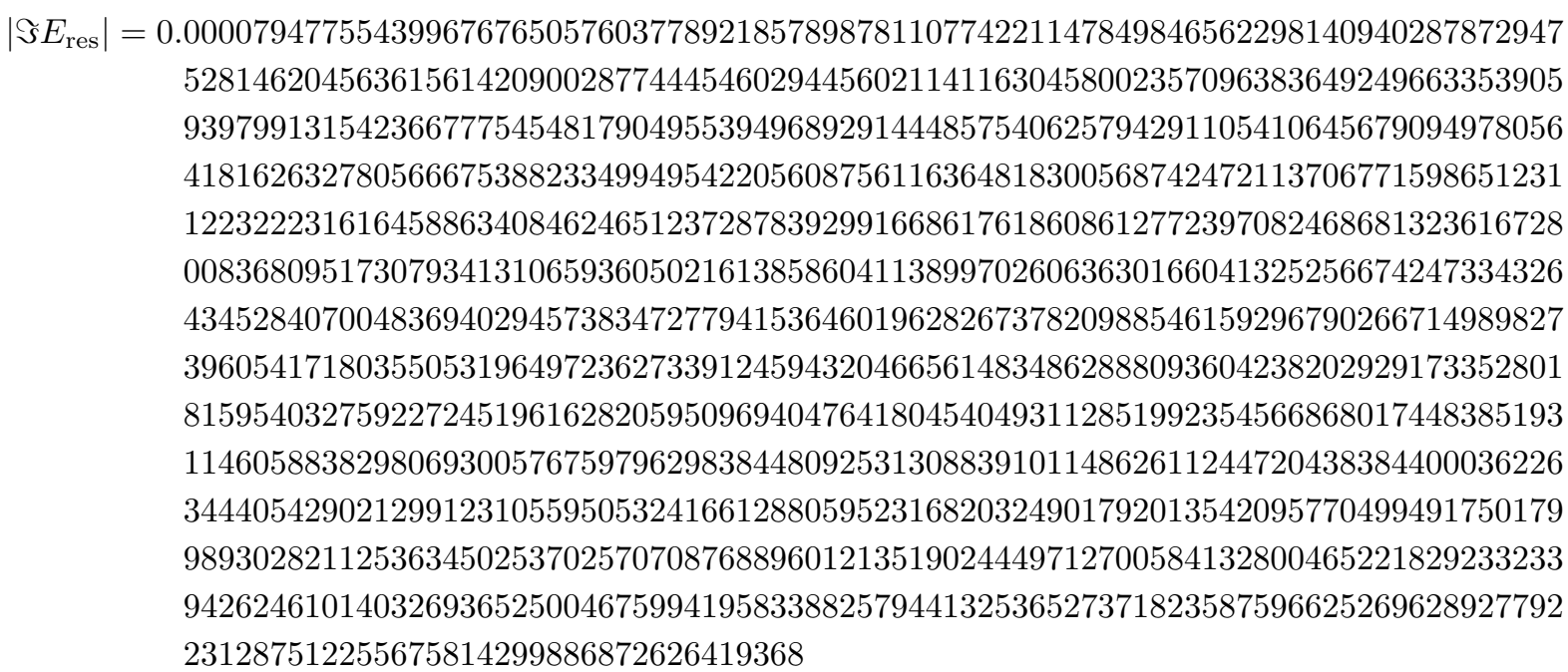

There are also resonances among the odd solutions that, as far as we know, have not been reported so far. For 


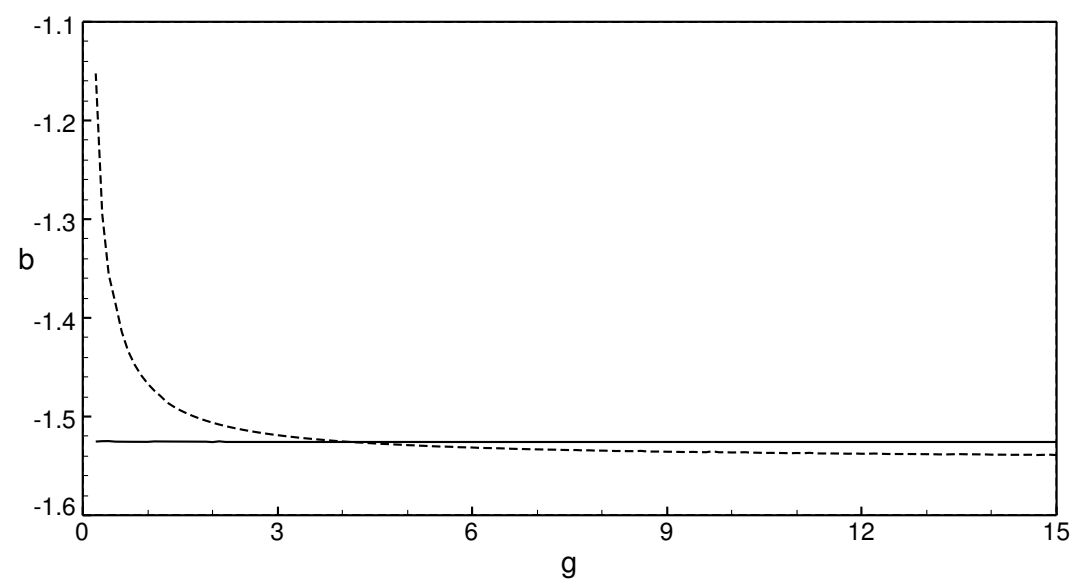

Figure 3. Slope $b(g)$ for the lowest even bound state (dashed line) and resonance (solid line) of the oscillator 14.

example, from determinants of dimension $D \leq 675$ we have estimated

$$
\begin{aligned}
\Re E= & 261567434444732550869411505135245734080470814119820813364017253899303022317155434 \\
& 31391542639715932621127326167835416895521493397749245764591162235274203164591933 \\
& 37244178082714412489418172286638540171259856180564528065416001947539967118936646 \\
& 75571877202323193379545799865521628801842268028131214215002900102213105491896812 \\
& 63767311711861097761090862732339313659663532602566940509690710619685623673577427 \\
& 02051685651830157091563631235768241343388226233422149668046828337636970321537470 \\
& 63887197465955131025788601409986649525116758890729464265922333123256268369696059 \\
& 26941420850055176884146238393937196844266014963704437945590546005662856976368611 \\
& 00640491298239429318015921498341474779363074158208007782572407910054254871310091 \\
& 68973174851641282036957946732252836879151952555347108138232897278070434422830026 \\
& 26704068364463061804095353910257042206327201629122973971604907355532903654161470 \\
& 19439703881706172720156058384564059563205471991977312223713679619282236509718612 \\
& 52126293985829302685817040822296771556201493457627526395925393874890357857758914 \\
& 87383866271
\end{aligned}
$$

and

$$
\begin{aligned}
|\Im E|= & 0.01210300605494968941953209254767687990290906183125611460665539748357431001508023 \\
& 85800465110061978821958452802312257429199680274874976714780325247533274025385051 \\
& 78880120535081995629925326152262013101282905412439967483243664748674923025125583 \\
& 00454126258993517009810558255025650762548260826491229849682682236927325028722181 \\
& 10911763777690357826524770782300369066474079388696220057742156320915294160429729 \\
& 01294409355539289307179903232721645370003682922827262095476379354302765153737115 \\
& 51880437155380856923219516679773451544032806175085515778612944592637256659887303 \\
& 30512036322261513744864521271324704804249317847540296878526243141260350713152579 \\
& 08326500268541345099397960617177832968775259890292555657102142040050150226184141 \\
& 73159023284305913490500706092689513886545759177324808900693017255028438277583325 \\
& 25877330744338536629042846929583130852297856757526856279666216561614392371093267 \\
& 54690511115693585022173938512605471651051770646269662062872998357747210540745535 \\
& 26126483662119100873839728146718246141274682165252936046723308704728026198914481 \\
& 33285093213
\end{aligned}
$$

The rate of convergence of the RPM for the bound states and the resonances may be different. One way of monitoring the rate of convergence is to calculate $\log \left|E^{[D]}-E^{[D-1]}\right|$ which is a straight line when the rate of convergence is exponential. In the present case we fit $y(D)=a+b D$ to $\log \left|E^{[D]}-E^{[D-1]}\right|$ and obtain $b(g)$ for several values of $g$. The results for the lowest even bound state and resonance are shown in Figure 3 That figure clearly shows that the 
rate of convergence for the resonance is almost constant whereas it decreases with $g$ for the bound state. As a result it is possible to obtain the resonance for small values of $g$, say $g<1$, more accurately by means of determinants of similar and even lesser dimension. However, this advantage is counterbalanced by the fact that the mathematical operations require more CPU time when complex numbers are involved.

In order to carry out the necessary arithmetic of complex numbers with arbitrarily high precision we resorted to the GNU MPC C library[10] and to the recurrence relation:

$$
H_{D}^{d}=\frac{H_{D-1}^{d} H_{D-1}^{d+2}-\left(H_{D-1}^{d+1}\right)^{2}}{H_{D-2}^{d+2}}
$$

for a fast calculation of the Hankel determinants.

\section{Conclusions}

Throughout this paper we tried to stress two points. The first one is that the RPM can yield eigenvalues of remarkably accuracy if the algorithm is programmed judiciously. To this end we have calculated the lowest eigenvalues of the oscillators 12 (with $\lambda=1$ ) and 13 with greater accuracy than those reported by Gaudreau et al[1] and Trott [2], respectively.

The second point is that only one RPM quantization condition applies to bound states and resonances. To illustrate it we calculated the resonances for two models with great accuracy. One of them is an ordinary resonance embedded in the continuum and other one is some kind of strange resonance appearing in the point spectrum close to the ground state. Present results for the lowest resonance in the discrete spectrum of the three-well potential (14) give support to the conjecture that the analytical WKB formula for the resonance width derived several years ago 8 may not be correct. Present results improve considerably upon those reported earlier by Killingbeck 9 and Fernández[2]. It is not clear to us whether the DESCM[1] or the power series approach [2] may also be applied to resonances without considerable modification of the calculation algorithms.

It is not our purpose to criticise the DESCM which is clearly a powerful algorithm as already proved by the remarkable calculations carried out by Gaudreau et al[1] on a wide variety of one-dimensional models. We just wanted to draw attention to some advantages of the RPM that have been overlooked in the discussion of the method carried out by those authors.

\section{REFERENCES}

[1] Gaudreau, P. J., Slevinsky, R. M., and Safouhi, H., Computing energy eigenvalues of anharmonic oscillators using the double exponential Sinc collocation method, Ann. Phys. 360, 2015, p. 520-538.

[2] Trott, M., High-Precision Value for the Quartic Anharmonic Oscillator Ground State. arXiv:quant-ph/0012147.

[3] Fernández, F. M., Ma, Q., and Tipping, R. H., Tight upper and lower bounds for energy eigenvalues of the Schrödinger equation, Phys. Rev. A 39, 1989, p. 1605-1609.

[4] Fernández, F. M., Ma, Q., and Tipping, R. H., Eigenvalues of the Schrödinger equation via the Riccati-Padé method, Phys. Rev. A 40, 1989, p. 6149-6153.

[5] Fernández, F. M., Direct Calculation of Accurate Siegert Eigenvalues, J. Phys. A 28, 1995, p. 4043-4051.

[6] Fernández, F. M., The accurate calculation of resonances in multiple-well oscillators, J. Phys. A 41, 2008, p. 065202.

[7] Fernández, F. M., Tunnel resonances for one-dimensional barriers, Chem. Phys. Lett. 281, 1997, p. 337-342.

[8] Benassi, L., Graffi, S., and Grecchi, V., Multiple well anharmonic oscillators and perturbation theory, Phys. Lett. B 82, 1979, p. 229-232.

[9] Killingbeck, J. P., A Hill-series method for resonances, J. Phys. A 40, 2007, p. 9017-9024.

[10] Andreas, E., Gastineau, M., Théveny, P., and Zimmerman, P., A library for multiprecision complex arithmetic with exact rounding. http://mpc.multiprecision.org/. 